\title{
A biomechanical model via Kane's equation - solving trunk motion with load carriage
}

\author{
Sharifah Alwiah AbdulRahman ${ }^{1 *}$, Azmin Sham Rambely ${ }^{2}$, Rokiah Rozita Ahmad ${ }^{2}$ \\ ${ }^{1}$ UTM Razak School of Engineering and Advanced Technology, \\ Universiti Teknologi Malaysia International Campus, Kuala Lumpur, Malaysia \\ ${ }^{2}$ Centre of Mathematical Science, Faculty of Science \& Technology, Universiti Kebangsaan \\ Malaysia, Bangi, Selangor, Malaysia \\ *Corresponding author, E-mail: shalwiah@ic.utm.my
}

\begin{abstract}
Carrying a heavy backpack has become a phenomenon among school children and adults. A review of the literature on the effect of carrying backpack loads on the trunk backward and forward lean in children and adults showed that with a load of even less than $15 \%$ of body weight, children have to walk with a forward lean of the trunk, whereas adults are in a forward lean position only with a backpack loads of more than $27 \%$ of their body weight. Due to these biomechanical studies, a mathematical model of the upper body representing trunk and head inclination angles is developed in this paper. The model was a two-link planar of rigid bodies in two-dimensional space. The objectives of this study were to investigate the motion of the trunk and also to develop the mathematical model so that the joint forces and moments acting on the body can be determined. The equations of motion for this model were written based on Kane's dynamical equation of motion.
\end{abstract}

Keywords : Backpack, load carriage, mathematical model, Kane's equation.

\section{INTRODUCTION}

Backpacks are a common form of load carriage among military personnel, hikers and school children of all ages. However, when a backpack is positioned at the back of the body, it changes body posture because of the adjustment of the center of gravity. In response to this adjustment, the body naturally has to shift either backward or forward to counterbalance the load of the backpack.

Many researchers have performed a biomechanical analysis on backpack load carriage in school children and adults. Some of the biomechanics factors on the effects of carrying heavy backpack loads included the inclination of the trunk and head and the curvature of the spine. Trunk forward lean is defined as the angle between a vertical line and a line created by connecting points at the greater trochanter and the acromion process (Goh et al., 1998). There are articles that have reported on the effects of carrying backpack loads on trunk inclination in adults (Goh et al., 1998; Knapik et al., 1996; Al-Khabbaz et al., 2008; Bloom and Woodhull-McNeal, 1987) and children (Garciaguirre et al., 2007; Rahman et al., 2009; Hong and Brueggemann, 2000; Hong and Cheung, 2003; Li and Hong, 2001; Shasmin et al.,
2007; Pascoe et al., 1997). However, studies on the effects of carrying backpack load on adults have

been done while walking (Goh et al., 1998) and also while standing (Al-Khabbaz et al., 2008; Bloom and Woodhull-McNeal, 1987). Different methods of carrying backpack load have also been used to investigate the inclination of the trunk, including carrying the load at the front, at the back and at the side of the body, carrying a backpack with one or two straps and carrying a frame backpack.

Studies on the effect of carrying heavy backpacks in children have been done on children as young as 14month-old infants. To examine the ways 14-monthold infants cope with the additional balance control problems induced by load carriage, Garciaguirre et al. (2007) analyzed infants' responses to various load conditions at the front and the back of their bodies. It was shown that even though infants tend to walk with a forward lean of the trunk or upright with no load, they walked with a backward trunk lean when carrying a load of $15 \%$ of their body weight at the back of their bodies, and they tended to walk forward when carrying a load of $15 \%$ of body weight at the front of their bodies. In this case infants 
accommodated the load carriage to control the balance of the body while walking.

a forward lean of the trunk when carrying a backpack load from $0 \%$ to $20 \%$ of their body weight. The trunk inclination angle increased more than $5^{\circ}$ with loads of $15 \%$ and $20 \%$ of the body weight compared with the $0 \%$ and $10 \%$ load conditions. The result is in agreement with the findings of Hong and Brueggemann, (2000) who examined the gait pattern, heart rate and blood pressure in 15 boys aged 10 years old, carrying backpacks weighing $0 \%$ (as a control), $10 \%, 15 \%$ and $20 \%$ of their body weight while walking on a treadmill. A significant difference was found between the $0 \%$ and $15 \%$ or $20 \%$ load conditions, with a significant increase in trunk forward lean.

Another study on trunk posture responses to backpack load during level walking in children was conducted by Hong and Cheung, (2003). Eleven primary school boys from 9 to 10 years old participated in the experiment. The subjects were asked to carry backpack loads of $0 \%, 10 \%, 15 \%$ and $20 \%$ of their body weight while walking around a perimeter of a basketball court, a total distance of $1978 \mathrm{~m}$. The results showed that there was a significant increase in the trunk inclination angle for the $20 \%$ load compared to the $0 \%, 10 \%$ and $15 \%$ of body weight conditions. The positive degree of the trunk inclination angle for each backpack weight means that the children walked with a forward trunk lean in all load conditions. Li and Hong, (2001) investigated the changes of trunk position and breathing pattern in children aged $10.31 \pm 0.26$ years old when carrying different weights of school bags while walking on a treadmill for 20 minutes. Twentyfive boys participated in four walking trials in which they carried school bags of $0 \%, 10 \%, 15 \%$ and $20 \%$ of their body weight. The results showed that the $20 \%$ load condition induced a significant increase in trunk forward lean, decreased trunk movement range and increased respiratory frequency.

Shasmin et al. (2007) conducted a study on trunk inclination among primary students aged between 9 and 11 years old when carrying backpack loads of $0 \%, 10 \%, 15 \%$ and $20 \%$ of their body weight. A significant increase in trunk forward lean was observed at a load of $15 \%$ of body weight, which suggested that the safe load carriage for children under 12 years old cannot exceed $15 \%$ of body weight.
However, a study by Rahman et al. (2009) showed that children aged $6.50 \pm 0.25$ years old walked with

Pascoe et al. (1997) investigated the effect of different methods of carrying schoolbags on gait and postural changes in 10 students aged 11 to 13 years old. In their study, the subjects participated in four different conditions: without a bag, with a one-strap backpack, with a two-strap backpack and with a onestrap athletic bag. The bags were loaded with $17 \%$ of their mean body weight. The students' trunk angle indicated a significant forward lean during both backpack trials. This forward lean was the result of subjects shifting their trunk segment forward to counterbalance the load of the backpack.

However, only a few studies on the effect of carrying a backpack on trunk posture (relating to trunk backward and trunk forward lean) have been conducted on adults. Goh et al. (1998) studied the load carriage of young adults aged $19 \pm 1.1$ years during walking, with three different load conditions: $0 \%$ (as a control), $15 \%$ and $30 \%$ of body weight. In the control condition (no load), it was found that subjects walked with a backward trunk lean throughout the gait cycle, with a mean trunk angle of $-8.38^{\circ}$. While walking with a load of $15 \%$ of the body weight, the mean trunk angle was reduced to about $-0.57^{\circ}$ in extension. However, walking with a load of $30 \%$ of the body weight resulted in a mean trunk angle of about $+4.26^{\circ}$, which implied that the subjects walked with a forward trunk flexion posture during this load condition.

Another study on adults was reported by Al-Khabbaz et al. (2008). In this study, 19 males aged $21 \pm 3$ years were asked to stand in four modes: unloaded standing, $10 \%, 15 \%$ and $20 \%$ of body weight load. Unloaded standing served as a reference to the three different load modes. The results of the changes in trunk posture for all three conditions of backpack load showed that the trunk inclined backward $-3.37^{\circ}$, $3.02^{\circ}$ and $-3.90^{\circ}$ during $10 \%, 15 \%$ and $20 \%$ of the body weight load conditions, respectively.

Bloom and Woodhull-McNeal, (1987) examined the changes in body posture induced by two types of frame backpack: the internal-frame pack and the external-frame pack on nine women and seven men between $19-25$ years old. The study examined how carrying each pack with a load of an average of $27 \%$ of body weight changed the body posture compared with the typical standing posture. The results showed that both types of packs caused subjects to lean 
forward, even though the body was bent forward significantly more under the internal-frame pack.
Loads used in biomechanical studies in children range from $0 \%$ to $20 \%$ of the body weight, whereas for adults, the load range was from $0 \%$ to $30 \%$ of

Table 1 Biomechanics studies on the effect of backpack loads on trunk backward and forward lean

\begin{tabular}{|c|c|c|c|c|c|c|c|c|}
\hline Study & Age (Years) & Sex & $\mathrm{N}$ & Distance & \multicolumn{4}{|c|}{$\begin{array}{c}\text { Load Carriage (\%BW) } \\
\text { Backward/Forward Lean }\end{array}$} \\
\hline $\begin{array}{l}\text { Garciaguirre et al. } \\
(2007)\end{array}$ & 14 months & M\&F & 27 & $-(W)$ & $\begin{array}{c}0 \\
\text { TFL }\end{array}$ & $\begin{array}{l}15 \\
\text { TBL }\end{array}$ & & \\
\hline $\begin{array}{l}\text { Rahman et al. } \\
(2009)\end{array}$ & $6.50 \pm 0.25$ & $\mathrm{M}$ & 2 & $8 \mathrm{~m}(\mathrm{~W})$ & $\begin{array}{c}0 \\
\text { TFL }\end{array}$ & $\begin{array}{l}10 \\
\mathrm{TFL}\end{array}$ & $\begin{array}{l}15 \\
\mathrm{TFL}\end{array}$ & $\begin{array}{l}20 \\
\text { TFL }\end{array}$ \\
\hline $\begin{array}{l}\text { Hong and Cheung } \\
(2003)\end{array}$ & $9.43 \pm 0.51$ & $\mathrm{M}$ & 11 & $1978 \mathrm{~m}(\mathrm{~W})$ & $\begin{array}{r}0 \\
T F L\end{array}$ & $\begin{array}{l}10 \\
\text { TFL }\end{array}$ & $\begin{array}{l}15 \\
\mathrm{TFL}\end{array}$ & $\begin{array}{l}20 \\
\mathrm{TFL}\end{array}$ \\
\hline $\begin{array}{l}\text { Hong and Brueggemann } \\
(2000)\end{array}$ & 10 & $\mathrm{M}$ & 15 & - TM & $\begin{array}{c}0 \\
\mathrm{TFL}\end{array}$ & $\begin{array}{l}10 \\
\mathrm{TFL}\end{array}$ & $\begin{array}{l}15 \\
\mathrm{TFL}\end{array}$ & $\begin{array}{l}20 \\
\mathrm{TFL}\end{array}$ \\
\hline Shasmin et al. (2007) & $10.28 \pm 0.72$ & $M$ & 7 & $5 \mathrm{~m}(\mathrm{~W})$ & $\begin{array}{c}0 \\
\text { TFL }\end{array}$ & $\begin{array}{l}10 \\
\text { TFL }\end{array}$ & $\begin{array}{l}15 \\
T F L\end{array}$ & $\begin{array}{l}20 \\
\mathrm{TFL}\end{array}$ \\
\hline Pascoe et al. (1997) & $11-13$ & $\mathrm{M}$ & 10 & $-(S \& W)$ & $\begin{array}{c}0 \\
\text { TFL }\end{array}$ & $\begin{array}{l}17 \\
\mathrm{TFL}\end{array}$ & & \\
\hline Goh et al. (1998) & $19.90 \pm 1.10$ & $\mathrm{M}$ & 10 & $-(W)$ & $\begin{array}{c}0 \\
\mathrm{TBL} \\
\end{array}$ & $\begin{array}{l}15 \\
\mathrm{~TB}\end{array}$ & $\begin{array}{l}30 \\
\text { TFI }\end{array}$ & \\
\hline $\begin{array}{l}\text { Al-Khabbaz et al } \\
(2008)\end{array}$ & $21 \pm 3$ & M & 1 & $-(S)$ & $\begin{array}{l}10 \\
\mathrm{TBL}\end{array}$ & $\begin{array}{l}15 \\
\mathrm{TBL}\end{array}$ & $\begin{array}{l}20 \\
\mathrm{TBL}\end{array}$ & \\
\hline $\begin{array}{l}\text { Bloom and Woodhull- } \\
\text { McNeal (1987) }\end{array}$ & $19-25$ & M\&F & 16 & $-(S)$ & $\begin{array}{l}0 \\
\mathrm{TBL} \\
\end{array}$ & $\begin{array}{l}27 \\
\mathrm{TFL}\end{array}$ & & \\
\hline
\end{tabular}

$\mathrm{BW}=$ body weight; $\mathrm{TFL}=$ trunk forward lean; $\mathrm{TBL}=$ trunk backward lean; $\mathrm{M}=$ male; $\mathrm{F}=$ female;

$\mathrm{W}=$ walking; $\mathrm{S}=$ standing; $\mathrm{TM}=$ treadmill walking

body weight. As the backpack load of the children increases, the degree of the trunk forward lean also increases. Adults tended to stand or walk with a forward lean of the trunk only when the load was $27 \%$ of their body weight.

Table 1 shows that without any load, children aged from 14 months to 13 years are already in the forward lean position, which is in contrast with adults, who are in the backward lean position when carrying no load. Furthermore, by comparing the results of children and adults, it seems that children have to lean forward while carrying backpack load, regardless of its percentage of the body weight, whereas adults only lean forward when the load increases to $30 \%$ of the body weight when walking (Goh et al., 1998) and $27 \%$ of body weight when standing (Bloom and Woodhull-McNeal, 1987), which shows that the load condition is heavy for adults. Thus if these load conditions are heavy for adults and they have to lean forward to counterbalance the load at the back of their bodies, then carrying loads of $10 \%, 15 \%$ and $20 \%$ of the body weight will be heavier for children because at these load conditions, they also tend to walk with a forward flexion. In addition, this forward flexion will induce greater postural effects on children and teenagers because the musculoskeletal system is vulnerable during these growing years.

Studies also proved that carrying a backpack load leads to a forward lean of the head (Pascoe et al., 1997; Chansirinukor et al., 2001). It was reported that carrying a load of $15 \%$ of the body weight while standing increased forward head posture, which supports the hypothesis that heavy loads have a significant effect on postural alignment (Chansirinukor et al., 2001). These changes in alignment of the neck can produce a strain in the cervical joints and soft tissue as well as imbalance muscle performance. This can cause pain in the cervical, upper thoracic and shoulder regions (Voll and Klimt, 1977).

Based on these observations and knowing that increasing backpack load can cause an increase in the forward inclination of the trunk and the head, it is essential to study the human upper limbs involving the trunk and the head. Because measuring the trunk inclination angle has been one of the methods used to determine the effects of backpack loads on the changes of posture in children and adults (Rahman et al., 2009), it is important to develop a mathematical 
model so that the joint forces and moments acting on the body can be determined by substituting the values of the various parameters, such as the trunk and head inclination angles.

Model of the Upper Limb: The study of the human upper limb plays an important role in human movement. However, most studies of the upper limb deal with the arms and shoulders. A musculo-skeletal model of the human arm involving the humerus, ulna, radius and hand was presented by Pennestri et al. (2007), whereas Flash and Hogan (1985) presented a mathematical model of the coordination of arm movements.

Studies of the upper limb involving the trunk and head are rare in the literature, especially those using Kane's equation. The model in this study represents the upper segments of a trunk and head in a sagittal plane. Equations of motion for this model are written based on Kane's equation. Kane's equation is a dynamic formulation of multi-body systems based upon Langrange's form of d'Alembert's principle, also known as the principle of virtual power developed by Kane and Levinson. Kane's method introduced the concepts of generalized speed, partial velocity, partial angular velocity, generalized active and generalized inertia forces (Kane and Levinson, 1985). With Kane's equation, non-working constraint forces between adjoining bodies are automatically eliminated in the analysis and thus simplify the dynamics equations of motion (Yamaguchi, 2006). However, Kane's method has been used mostly in the study of robotic systems (Meghdari and Fahimi, 1999; Samak and Gupta, 1998; Tarn and Yang, 1997), and a few researchers used Kane's method for studying an impact in a multi-body system (Chang and Huston, 1995; Huang, 1998). Komistek et al. (1998) used Kane's method of dynamics to describe a mathematical model for defining the joint reaction forces of the lower extremities.

Ariff and Rambely (2008) also used Kane's method for the upper limbs, but for determining the torques of the joints of the shoulder, elbow and wrist during jumping in badminton smash. Thus, the aim of this study is to develop a mathematical planar model of the biomechanical linkages of the upper limb of the human trunk and head by using Kane's method

\section{MATERIALS AND METHODS}

Biomechanical Model of Trunk Inclination: In this study, a mathematical model is designed in twodimensional space to view the inclination angle of the trunk and the head. Figure 1 shows a skeletal model of the upper extremities of a human body in a sagittal plane. The two degrees of freedom of the human-body model are developed using Kane's dynamics equation of motion. The model of the upper segments is a twolink planar kinematic chain. Rigid link $A$ represents the trunk, and rigid link $B$ is for the head. There are two hinge joints, one at the sacrum and the other at the neck of the human-body model. The movements of the body can be described in terms of two generalized coordinates $q_{1}$ and $q_{2}$, representing the trunk and head inclination angles, respectively. Figure 2 is a free body diagram of the model showing the forces acting at each angle. The symbols used in this model are:

$$
\begin{aligned}
& A^{*}, B^{*}=\text { mass centers of bodies } A \text { and } B \\
& \ell_{A}, \ell_{B}=\text { lengths of segments } A \text { and } B \\
& \rho_{A}, \rho_{B}=\text { distances of the centers of mass from their }
\end{aligned}
$$
proximal ends

$$
\begin{array}{ll}
\vec{n}_{1}, \vec{n}_{2}, \vec{n}_{3}, \vec{a}_{1}, \vec{a}_{2}, \vec{a}_{3}, \vec{b}_{1}, \vec{b}_{2}, \vec{b}_{3}=\text { mutually orthogonal unit vectors } \\
q_{1} \quad=\text { trunk angle } \\
q_{2} \quad=\text { head angle } \\
\vec{v} \quad=\text { linear velocity } \\
\vec{a} \quad=\text { linear acceleration } \\
\vec{\omega} \quad=\text { angular velocity } \\
\vec{\alpha} \quad=\text { angular acceleration } \\
g \quad=\text { acceleration of gravity }
\end{array}
$$

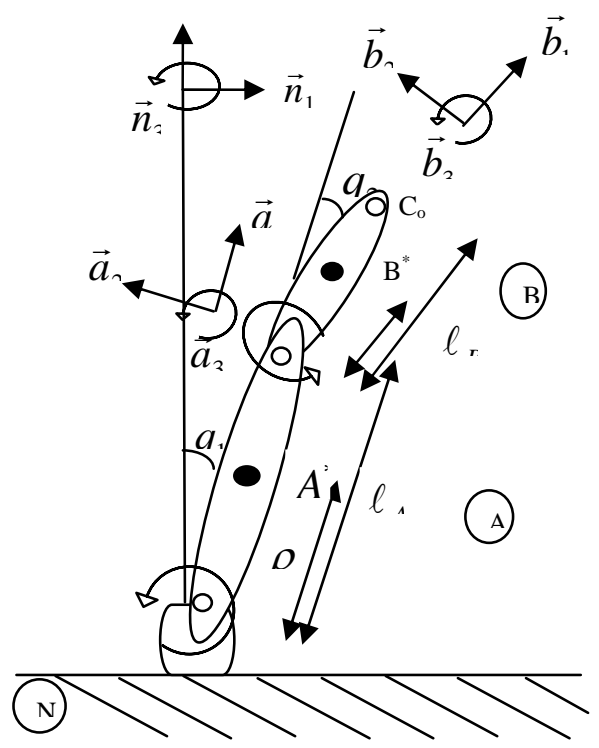

Figure 1: A model of two segments. $A$ represents the trunk, and $B$ represents the head. 


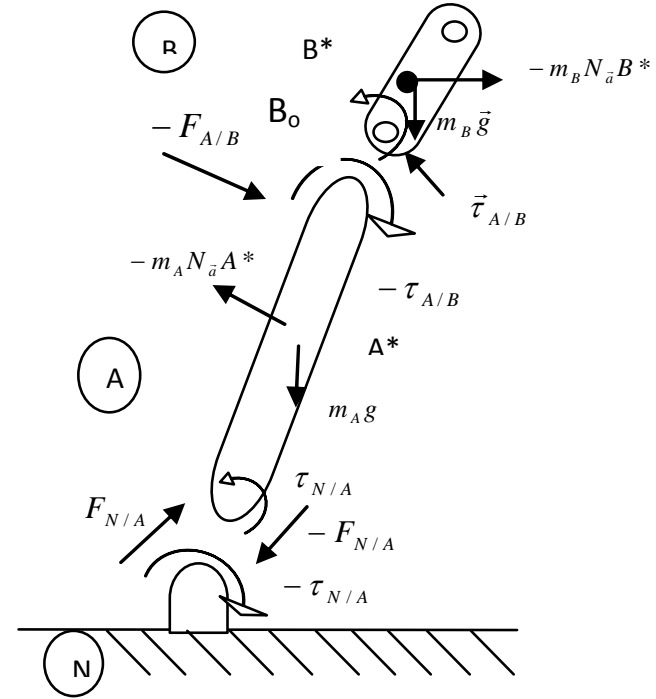

Figure 2: A free body diagram showing the forces acting at each angle

The upper body model in Figure 1 has two degrees of freedom featuring the trunk and the head. The kinematic equations are constructed using local reference frames $N, A$ and $B$ affixed to the body. The equation is created using a direction cosines table that can be obtained from the dot product of the unit vectors of each reference frame. This will produce linear velocities, linear accelerations, angular velocities and angular accelerations. The angular velocities of bodies $A$ and $B$ relative to reference frame $N$ are determined to be

$N_{-\omega} A=\dot{q}_{1} \overrightarrow{a_{3}}$

$N_{\vec{\omega}} B=\left(\dot{q}_{1}+\dot{q}_{2}\right) \vec{b}_{3}$

By differentiating (1) and (2), the angular accelerations of bodies $A$ and $B$ with respect to reference frame $N$ are determined to be

$N_{\vec{\alpha}} A=\ddot{q}_{1} \overrightarrow{a_{3}}$

$N_{-\alpha} B=\left(\ddot{q}_{1}+\ddot{q}_{2}\right) \overrightarrow{b_{3}}$

Using vector cross products, the velocities and accelerations of mass locations and at the joints with respect to reference frame $N$ can be obtained:

$$
\begin{aligned}
& N_{\vec{v}} A_{o}=0 \\
& N_{\vec{v}} A^{*}=\rho_{A} \dot{q}_{1} \vec{a}_{2} \\
& N_{\vec{v}} B_{o}=\ell_{A} \dot{q}_{1} \vec{a}_{2}
\end{aligned}
$$

$$
\begin{aligned}
& N_{\bar{v}} B^{*}=\ell_{A} \dot{q}_{1} \vec{a}_{2}+\rho_{B}\left(\dot{q}_{1}+\dot{q}_{2}\right) \vec{b}_{2} \\
& N_{\bar{v}} C_{o}=\ell_{A} \dot{q}_{1} \vec{a}_{2}+\ell_{B}\left(\dot{q}_{1}+\dot{q}_{2}\right) \vec{b}_{2}
\end{aligned}
$$

The accelerations of mass location and at the joint are given as

$$
\begin{aligned}
& N_{\vec{a}} A^{*}=-\rho_{A} \dot{q}_{1}{ }^{2} \vec{a}_{1}+\rho_{A} \ddot{q}_{1} \vec{a}_{2} \\
& N_{\vec{a}} B_{0}=-\ell_{A} \dot{q}_{1}^{2} \vec{a}_{1}+\ell_{A} \ddot{q}_{1} \vec{a}_{2} \\
& N_{\vec{a}} B^{*}=-\ell_{A} \dot{q}_{1}{ }^{2} \vec{a}_{1}+\ell_{A} \ddot{q}_{1} \vec{a}_{2}-\rho_{A}\left(\dot{q}_{1}+\dot{q}_{2}\right)^{2} \vec{b}_{1}+\rho_{B}\left(\ddot{q}_{1}+\ddot{q}_{2}\right) \vec{b}_{2}
\end{aligned}
$$

The kinematical equations are completed when the velocities of all points with forces acting through them are known, and all accelerations of mass centers are known as well.

\section{Kane's Method}

The angular velocities of bodies $A$ and $B$ with respect to reference frame $N$ can be factored in the form of generalized speeds $u_{1}$ and $u_{2}$, where $u_{i} \equiv \dot{q}_{i}(i=1,2)$ (for 2 degrees of freedom in the human-body model) to obtain the partial angular velocity of bodies $A$ and $B$ in $N$.

Then the quantities called partial angular velocities and partial velocity vectors must be generated directly from the expressions for angular velocity and velocity. The partial angular velocities are important quantities to define bodies that rotate in response to applied torques. The generalized active and inertial forces are then produced. The $n$ generalized active forces $F_{r}$ for a system $S$ in reference frame $N$ with $n$ degrees of freedom, $v$ points at which forces act and $\mu$ rigid bodies upon which torques act are defined as

$F_{r}=\sum_{i=1}^{v} N_{\vec{v}_{r}} P_{i} \cdot \vec{R}_{i}+\sum_{j=1}^{\mu} N_{\bar{\omega}_{r}} B_{j} \cdot \vec{\tau}_{j}$

For $r=1,2, \ldots, n, \vec{R}_{i}$ is the sum of all distance and body forces (except for inertial forces) acting at each point $P_{i}(i=1,2, \ldots, v)$ and $\vec{\tau}_{j}$ is the torque acting on each body $B_{j}(j=1,2, \ldots, \mu)$.

The $n$ generalized inertia forces $F_{r}{ }^{*}$ for a system $S$ with $n$ degrees of freedom in reference frame $N$ are defined as

$$
F_{r}^{*}=\sum_{i=1}^{v} N_{\vec{v}_{r}} P_{i}^{*} \cdot \vec{R}_{i}^{*}+\sum_{j=1}^{\mu} N_{\vec{\omega}_{r}} B_{j} \cdot \vec{\tau}_{j}^{*}
$$


For $r=1,2, \ldots, n, \vec{R}_{i}^{*}$ is defined to be the inertial force acting at each mass center $P_{i}^{*}(i=1,2, \ldots, v)$,

$$
\vec{R}_{i}^{*}=-m_{P_{i}^{*}} N_{\vec{a}} P_{i}^{*}
$$

where $m_{P_{i}^{*}}$ is the mass concentrated at point $P_{i}^{*}$ and $N_{\vec{a}} P_{i}^{*}$ is the acceleration of $P_{i}^{*}$ in reference frame $N$. The variable $\vec{\tau}_{j}{ }_{j}$ is defined to be the inertial torque for body $B_{j}$ in $N,(j=1,2, \ldots, \mu)$

$$
\vec{\tau}_{j}^{*}=-I_{B} N_{\vec{\alpha}} B_{j}
$$

where $I_{B}$ is the moment of inertia of $B_{j}$ about an axis parallel to its angular acceleration $N_{\vec{\alpha}} B_{j}$ and passing through the center of mass of body $B_{j}$.

Using all the quantities from the kinematic equation, Kane's dynamics equations of motion can be obtained by adding up the generalized active forces and the generalized inertia forces and setting them equal to zero:

$$
\begin{aligned}
& {\left[\begin{array}{r}
\vec{a}_{3}\left(\tau_{N / A}-\tau_{A / B}\right)+\vec{b}_{3} \cdot \tau_{A / B} \\
\vec{b}_{3} \cdot \tau_{A / B}
\end{array}\right] } \\
= & {\left[\begin{array}{rr}
m_{A} \rho_{A}{ }^{2}+m_{B} \ell_{A}{ }^{2}+m_{B} \rho_{B}{ }^{2}+I_{A^{*}}+I_{B^{*}} & m_{B} \rho_{B}{ }^{2}+I_{B^{*}} \\
m_{B} \rho_{B}{ }^{2}+I_{B^{*}} & m_{B} \rho_{B}{ }^{2}+I_{B^{*}}
\end{array}\right]\left[\begin{array}{l}
\ddot{q}_{1} \\
\ddot{q}_{2}
\end{array}\right] } \\
& -\left[\begin{array}{cc}
-\rho_{A} \sin q_{1} & -\ell_{A} \sin q_{1}-\rho_{B} \sin q_{2} \cos q_{1}-\rho_{B} \cos q_{2} \sin q_{1} \\
0 & -\rho_{B} \sin q_{2} \cos q_{1}-\rho_{B} \cos q_{2} \sin q_{1}
\end{array}\right]\left[\begin{array}{c}
m_{A} g \\
m_{B} g
\end{array}\right] \\
& -\left[\begin{array}{c}
f_{1} \ell_{A}\left(-\cos q_{1}\right)+f_{1} \ell_{B}\left(\sin q_{1} \sin q_{2}-\cos q_{1} \cos q_{2}\right)+f_{2} \ell_{A} \sin q_{1}+f_{2} \ell_{B}\left(\sin q_{2} \cos q_{1}+\cos q_{2} \sin q_{1}\right) \\
f_{1} \ell_{B}\left(\sin q_{1} \sin q_{2}-\cos q_{1} \cos q_{2}\right)+f_{2} \ell_{B}\left(\sin q_{2} \cos q_{1}+\cos q_{2} \sin q_{1}\right)
\end{array}\right]
\end{aligned}
$$

Thus the dynamics equation (11) can be represented by inverse matrices:
These matrices can be used to investigate the motion of the trunk and determine the joint forces and moments acting on the body.

\section{DISCUSSION}

Load carriage is a daily task for soldiers, adult workers, college students and school children. Although many studies have been conducted to determine the effect of carrying a heavy backpack load in adults and children, the comparison between the forward and backward leans in adults' and children's trunks has not yet been reported. The effect of backpack loads on the trunk's forward and backward lean for adults and children in Table 1 shows that children have to lean forward while carrying backpack loads, regardless of the percentage of their body weight, whereas adults only lean forward when the load is at least $27 \%$ of their body weight.

This probably explains the findings that spinal ligaments and muscles are not fully developed until after the 16th year of life (Patrick, 2006). As we know, children experience rapid growth and have immature bony ossification. Compared to adults, their bones are particularly soft, and, as a result, they can easily bend. It is widely believed that the prolonged carriage of heavy backpacks will cause additional stress on the rapidly growing spine, especially in younger children. This will make them more prone to postural changes that will eventually 
lead to lower back problems. Thus, for younger children, who are still growing, the increase in load carriage will force them to lean forward to bring the center of gravity back over the base of support.

Thus, the study of the trunk inclination angle has become one of the methods used to determine the effects of backpack loads on the changes of posture in children and adults. In doing so, the present mathematical model provides a framework for modeling the planar biomechanical linkages of the upper limb of the human trunk and head by using Kane's method.

\section{CONCLUSION}

In this paper, an upper limb mathematical model has been presented by using Kane's dynamical equations of motion. From the model, the torque values are able to provide a description of trunk and head movements while walking with loads. This will enables us to determine the effects of backpack load on changes in posture in children especially and adults in general.

\section{REFERENCES}

Al-Khabbaz, Y.S.S.M., Shimada, T. and Hasegawa, M. (2008). The effect of backpack heaviness on trunklower extremity muscle activities and trunk posture. Gait and Posture. 28: 297-302

Ariff, F.H. and Rambely, A.S. (2008). Determination of torques at upper limb joints during jumping in badminton smash via Kane's method: Scientific Proceedings of the $26^{\text {th }}$ International Symposium on Biomechanics in Sports held at Seoul, Korea, pp. 7376

Bloom, D. and Woodhull-McNeal, A.P. (1987). Postural adjustments while standing with two types of loaded backpack. Ergonomics. 30(10): 1425 - 1430

Chang, C.C. and Huston, R.L. (1995). Computational methods for studying impact in multibody sytems. Computers \& Structures. 57(3): $421-425$

Chansirinukor, W., Wilson, D., Grimmer, K. and Dansie, B. (2001). Effects of backpacks on students: Measurement of cervical and shoulder posture. Australian J. of Physiology. 47: 110-116

Flash, T. and Hogan, N. (1985). The coordination of arm movements: An experimentally confirmed mathematical model. The J. of Neuroscience. 5(7): $1688-1703$

Garciaguirre J.S., Adolph, K.E. and Shrout, P.E. (2007). Baby carriage: Infants walking with loads. Child Development. 78( 2): $664-680$
Goh, J.H., Thambyah, A. and Bose, K. (1998). Effects of varying backpack loads on peak forces in the lumbosacral spine during walking. Clin. Biomechanics. 13(1):S26 - S31

Hong, Y. and Brueggemann, G.P. (2000). Changes in gait patterns in 10-year-old boys with increasing loads when walking on a treadmill. Gait and Posture. 11: 254 $-259$

Hong, Y. and Cheung, C.K. (2003). Gait and posture responses to backpack load during level walking in children. Gait and Posture. 17: $28-33$

Huang, S.C. (1998). Analysis of human body dynamics in simulated rear-end impacts. Human Movement Science. 17: $821-838$

Kane,T.R. and Levinson, D.A. Dynamics: Theory and Applications, McGraw-Hill, New York, 1985

Knapik, J., Harman, E. and Reynolds, K. (1996). Load carriage using packs: A review of physiological, biomechanical and medical aspects. App. Ergonomics. 27(3): 207-216

Komistek, R.D., Stiehl, J.B., Dennis, D.A., Paxson, R.D. and Soutas-Little, R.W. (1998). Mathematical model of the lower extremity joint reaction forces using Kane's method of dynamics. J. of Biomechanics. 31: $185-$ 189

Li, J.X. and Hong, Y.(2001). Changes of trunk position and breathing pattern in children walking under conditions of load carriage: Scientific Proceedings of the XIX International Symposium on Biomechanics in Sports held at San Francisco, pp. 177-179

Meghdari, A. and Fahimi, F. (1999). Dynamic modeling of multi-elastic body systems using Kane's method and congruency transformations. Technische Mechanik. 19(2): $127-140$

Pascoe, D.D., Pascoe, D.E., Wang, Y.T. Shim, D.M. and Kim, C.K. (1997). Influence of carrying book bags on gait cycle and posture of youths. Ergonomics. 40(6): $631-641$

Patrick,C. Spinal conditions, in: S.K. Campbell, D.W.V. Linden, and R.J. Palisano, Physical Therapy For Children, 3rd ed. Saundets, Philadelphia, PA., 2006, pp. $337-338$.

Pennestri, E., Stefanelli, R., Valentini, P.P. and Vita, L. (2007). Virtual musculo-skeletal model for the biomechanical analysis of the upper limb. J. of Biomechanics. 40: $1350-1361$

Rahman, S.A.S.A., Rambely, A.S. and Ahmad, R.R. (2009). A preliminary studies on the effects of varying backpack loads on trunk inclination during level walking. European J. of Scientific Research. 28( 2): $294-300$ 
Am. J. Sci. Ind. Res., 2011, 2(4): 678-685

Samak, S.M. and Gupta, K.C. (1998). Parametric uncertainty on manipulators dynamic. Mechanical Machine Theory. 33(7): 945 - 956

Shasmin, H.N., Abu Osman, N.A., Razali, R., Usman, J. and Wan Abas, W.A.B. (2007). Preliminary study of acceptable load carriage for primary school children. Biomed 06, Proceedings of the International Federation for Medical \& Biological Engineering held at Kuala Lumpur, 15, pp. $171-174$

Tarn, T.J. and Yang, S.P. (1997). Modeling and control for underwater robotic manipulators - An example: Proceedings of the 1997 IEEE International
Conference on Robotics and Automation held at Albuquerque, New Mexico, pp. 2166 - 2171

Voll, H.J. and Klimt, F. (1977). Translated by Theodridis, D. (1977): On strain in children caused by carrying schoolbags. (From Die beanspruchung des kindes durch die schultasche). Offentliche Gesundheitswesen. 39: $369-378$

Yamaguchi, G.T. Dynamic Modelling of Musculoskeletal Motion : A Vectorized Approach for Biomechanical Analysis in Three Dimensions, Springer, New York, 2006 